\title{
Führungs- und Managementkultur auf Station Maßnahmen für ruhigere, synchonisierte Abläufe
}

Welche Einrichtung hat in den letzten Jahren keinen massiven Stellenabbau im Pflegepersonal vollzogen? Gleichzeitig haben sich durch die demografischen Entwicklung und die modernen medizintechnischen Möglichkeiten, aber auch durch ein zunehmendes Gesundheitsbewusstsein der Bevölkerung die Patientenzahlen deutlich erhöht. Die damit einhergehende Fallzahlsteigerung wird nur zum Teil durch Verweildauerreduktionen aufgewogen. Was daraus resultiert ist eine massive Leistungsverdichtung in der stationären Krankenversorgung.

Wir widmen uns der Frage, wie in einem solchen Umfeld durch integrierte Organisationsmaßnahmen auf Station ruhigere Abläufe zu erreichen sind.

Die Arbeitsbelastung auf Station wird durch Fluktuation und einen hohen Krankheitsausfall innerhalb des Pflegedienstes weiter verschärft. Zeit für kurze Gespräche mit den Patienten oder FünfMinuten-Pausen werden unter diesem gewachsenen Zeit- und Leistungsdruck als kaum noch machbar empfunden. So sehen sich die Pflegekräfte teils nicht mehr in der Lage, die Werte des Krankenhauses zu leben und den Patienten die notwendige Fürsorge zukommen zu lassen. $\mathrm{Zu}$ sehr sind die Pflegenden damit beschäftigt, die anfallenden Arbeiten auf Station in der geforderten Zeit bzw. in ihrer Schicht zu bewältigen. Neben den pflegerischen Kerntätigkeiten umfasst dies nicht selten umfangreiche pflegefremde Tätigkeiten, wie z.B. Reinigungsund Transportdienste.

Unkoordinierte Entlassungen, neu aufgenommene Patienten, die auf den Fluren auf ihre Betten warten, Hektik und Multi- tasking unter den Mitarbeitern - ein unstrukturierter, unter den Berufsgruppen nur unzureichend synchronisierter Stationsablauf kann Folgen mit sich bringen, welche die Mitarbeiter zusätzlich unangemessen belasten und die Patientenversorgung beeinträchtigen.

\section{Ausgangslage: Defizite in der} berufsgruppenübergreifenden Ablauforganisation

Der Wandel hin zu geordneten Management-Strukturen, die Entlastung für alle Beteiligten mit sich bringen, bedarf einer gesunden Balance zwischen den Bedürfnissen von Patienten und Mitarbeitern und muss sich an der Effektivität von Stationsabläufen orientieren. Eine Lösung hierfür besteht darin, die Abläufe der verschiedenen Berufsgruppen und Organisationsbereiche an den Schnittstellen rund um die Patientenversorgung auf der Station eng miteinander zu verzahnen.
Für diesen Weg hat sich auch das Klinikum Gütersloh entschieden. Eine von der Geschäftsführung und der Pflegedirektion beauftragte Mitarbeiterbefragung auf einer Pilotstation hat folgende wesentlichen Engpässe im Stationsalltag identifiziert:

- Der Tagesablauf auf Station wird von den Mitarbeitern als nicht genügend strukturiert bewertet.

- Es existiert kein festgelegter Aufnahme- und Entlassprozess.

- Es existieren keine festgelegten Visitenzeiten oder feste Aufnahme- und Übernahmezeitfenster.

- Aufgrund dessen kommt es immer wieder zu Verzögerungen im Stationsablauf, sodass die anfallenden Aufgaben nicht in der regulären Dienstzeit erledigt werden können.

- Zeitziele und festgelegte Abläufe werden weder von Ärzten noch von der Pflege verbindlich eingehalten.

Die Ergebnisse der Mitarbeiterbefragung zeigten auch, dass der Wunsch nach einer Verbesserung der berufsgruppenübergreifenden Kommunikation besteht.

Die Aufnahmen und Entlassungen finden über den ganzen Tag verteilt statt und sind auf Station nicht synchronisiert (Abb. 1). Bei werktäglich 6,5 Aufnahmen auf Station (davon etwa $60 \%$ elektiv) stellt das einen erheblichen Belastungsfaktor über den ganzen Tagdienst dar.

Die Entlassungen beginnen wesentlich zu spät und ziehen sich bis in den Abend hinein, während die neuen Patienten bereits früh am Morgen aufgenommen werden. Hier zeigt sich ein deutlicher Handlungsbedarf in der organisatorischen Glättung von unnötigen Belastungsspitzen. Ad-Hoc-Entlassungen und unkoordinierte Aufnahmen führen zu $\subseteq$ erheblicher Unzufriedenheit bei Mitar능 beitern wie auch Patienten. Das Projektziel wurde dementsprechend plakativ dadurch beschrieben, die Aufnahmeund Entlasszeitenkurve zu vertauschen, am Ende also quasi die Farbverläufe genau umzukehren („aus Rot mach Blau, aus Blau mach Rot“). 
Management-Strukturen schaffen und die Aufbauorganisation auf Station stärken

Die Projektgruppe erarbeitete Verbindlichkeiten und Regelungen für den zukünftigen Stationsablauf und hielt diese schriftlich in einem Stationskonzept fest. Dieses hat zum Ziel, unnötige Arbeitsschritte zu reduzieren und Aufgaben sinnvoller zu verteilen. Das Potenzial hierfür ist immer wieder erstaunlich hoch.

Das Stationskonzept ist ein wichtiger Bestandteil des interpersonellen Wissensmanagements und der Wissenssicherung. Doch das ist und bleibt nur der Anfang. Wichtig ist es dann, die berufsgruppenübergreifenden Prozesse in die Alltagsroutine zu überführen. Sollen sich Mitarbeiter aller Berufs- und Hierarchiegruppen auf Station team- und tätigkeitsorientiert auf Augenhöhe begegnen, um die vereinbarten Absprachen und Prozesse tagtäglich zu leben, bedarf es des Wandels hin zu einer neuen Verantwortungskultur. Wesentliche Erfolgskriterien dafür sind die Definition klarer Führungsverantwortlichkeiten sowie die Neuverteilung der Aufgaben- und Kompetenzprofile [1]. Mitarbeiter müssen in Zeiten hoher Leistungsdichte und personeller Knappheit ganz besonders intrinsisch motiviert sein. Die Motivation der Pflegenden für die tägliche Arbeit erwächst erfahrungsgemäß eher aus den guten Teamstrukturen des Pflegeteams und meist weniger aus der Organisation bzw. dem Arbeitgeber.

Für die Einhaltung berufsgruppenübergreifender Managementstrukturen zu sorgen und Mitarbeitende individuell zu führen und damit intrinsische Motivation zu fördern - die Aufgabenstellung ist für die Führungskraft auf Station gewaltig. Weil die Aufgabe für die Funktionsfähigkeit des Gesamtsystems aber so wichtig ist, kommt der Auswahl der richtigen Führungskräfte eine tiefgreifende Bedeutung zu.

Am Klinikum Gütersloh wurde in der Vergangenheit eine stationsübergreifende Leitung etabliert, um erhöhte Ausgleichmöglichkeiten im Personalmanagement und fokussierter Freiräume für die tatsächliche Leitungstätigkeit zu schaffen. Der Erfolg des Organisationsentwicklungs-Projektes hängt von der Motivation, sich auf Neues einzulassen, und von der Selbstdisziplin der Beteiligten auf der Pilotstation ab. Dazu sind Führungsprä-

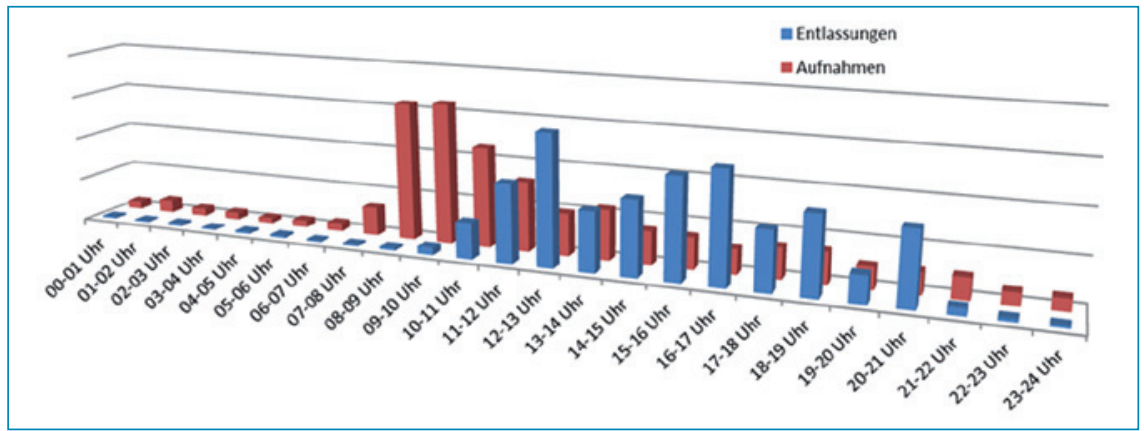

Abb. 1 Aufnahmen und Entlassungen auf Station, Stand März 2012.

senz und Führungskonsequenz zentrale Erfolgsfaktoren. Dementsprechend wurde der stationsübergreifende Leitungsansatz zur Begleitung des Veränderungsprozesses im Pilotzeitraum revidiert und der Pilotstation die Führungskraft vollumfänglich als Stationsleitung zur Verfügung gestellt. In Zeiten, in welchen gewohnte Routinen aufgebrochen und Veränderungen initiiert werden sollen, war dies eine von allen Beteiligten als notwendig erachtete Maßnahme.

Stationen sind geprägt von einer hohen Anzahl an Schnittstellen, Abteilungsgrenzen sowie Grenzen zwischen Berufsgruppen, die in sich hierarchisch strukturiert sind. Die Einführung erfolgreicher Stationsstrukturen erfordert daher eine Vernetzung von Verantwortungsbereichen und eine offene, prozessbezogene Aufgabenverteilung.

Für die Überwindung der seit Jahrzehnten existierenden Berufsgruppengrenzen auf kurzem Dienstweg und damit einhergehend für die Zusammenführung der wesentlichen Expertisen gibt es ein Konzept: das Managementtandem - ein Managementteam, das aus dem verantwortlichen Oberarzt und der Stationsleitung besteht. Ihnen obliegen die gemeinsame Prozessund Managementverantwortung sowie die Personalverantwortung jeweils für die ärztlichen und pflegerischen Mitarbeiter sowie das Unterstützungspersonal. Problemstellungen, die innerhalb der Station berufsgruppenübergreifend auftauchen, können im Managementteam gemeinsam berufsgruppenübergreifend reflektiert und direkt gelöst werden. Im Zusammenwirken der berufsgruppenübergreifenden Leitung existieren für den Bedarfsfall direkte disziplinarische Durchgriffmöglichkeiten. Ist die Rahmensituation der Verantwortungsübertragung im Vorfeld entsprechend geklärt, werden über die Struktur der Managementteams kurze Dienstwege eingerichtet.
Zuständigkeiten müssen stets von oben nach unten definiert und autorisiert werden, in diesem Fall sowohl durch den Chefarzt der betroffenen Abteilung als auch durch die Pflegedirektion. Stationsleitungen leben in ihrer Funktion als Führungskräfte Werte vor, vermitteln diese dem Pflegeteam und unterstützen das Team in ihrer Managementverantwortung im täglichen Bewältigen des Stationsablaufs. Durch die geteilte Managementverantwortung mit dem verantwortlichen Oberarzt soll gegenseitige Unterstützung erfahren werden, die berufsgruppenübergreifende Kommunikation sowie der wertschätzende Umgang miteinander nicht zuletzt auch vorgelebt werden. Engagement von Seiten der Führungskraft wird so für das Pflegeteam deutlich wahrnehmbar und mit Motivation und Einsatz belohnt [1]. Die mittlere Führungsebene steht im ständigen Kontakt mit den Mitarbeitern und fördert Stabilität und Mitarbeiterbindung.

\section{Umsetzung des Stationskonzepts: Vom Push- zum Pullprinzip}

Mit dem Aufbau des Managementteams sowie der Fertigstellung des Stationskonzepts fiel der Startschuss für die Umsetzungsphase des Stationskonzepts. Die Mitarbeiter wurden auf einer Mitarbeiterinformation über die wichtigsten Neuerungen im Stationsablauf informiert. Die Stationsleitung spielt hierbei eine zentrale Rolle. Gemeinsam mit dem organisationsverantwortlichen Oberarzt achtet sie auf die Einhaltung der Prozesse und sorgt für einen reibungslosen Ablauf auf Station. Dies ist insbesondere zu Beginn der Umsetzungsphase von großer Bedeutung, da die neu definierten Strukturen und Prozesse so lange auf ihre verbindliche Einhaltung hin überprüft werden, bis sie in einen Routineprozess übergegangen sind. Nach der Evaluation zur Einhaltung der Prozesse sind oftmals auch detaillierte Nachjustierungen möglich. 


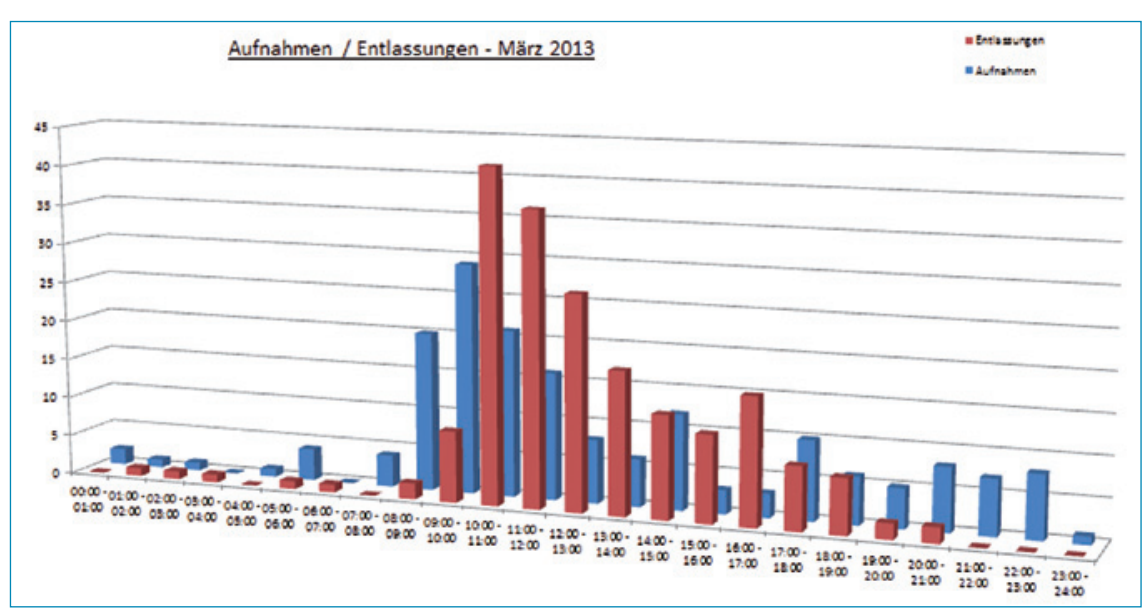

Abb. 3 Aufnahmen und Entlassungen auf Station, Stand März 2013.

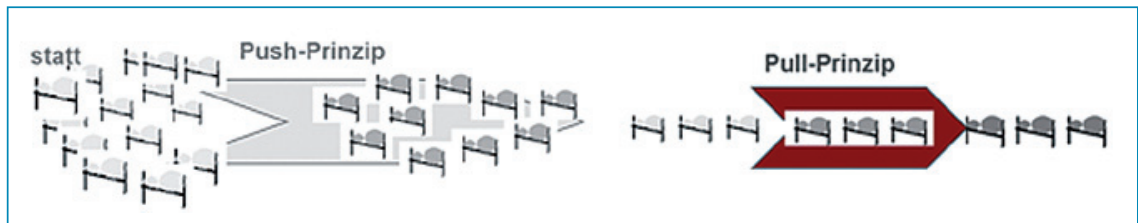

Abb. 2 Vom Push- zum Pull-Prinzip für geregeltere Abläufe auf Station.

Ein zentrales Ziel des Stationskonzeptes in Gütersloh war das Schaffen von mehr Ruhe auf Station durch gezieltes Management des Aufnahme-, Entlass- und Verweildauerprozesses. Hierfür wurden differenzierte Aufnahmeprozesse für Notfälle, elektive Patienten, Chemotherapie-Patienten sowie Verlegungen von Intensivstation ausgearbeitet. Die Einbestellung elektiver Patienten erfolgt nun gestaffelt zwischen 9 und 10 Uhr. Zudem wird spätestens 72 Stunden nach der Aufnahme das Entlassdatum geplant. Die Entlassplanung aus der Visite wird laufend in die EDV-gestützte Entlassliste übertragen und am Vortag der Entlassung in die Stationsgrafik eingetragen. Ziel ist es, dass $2 / 3$ aller Entlassungen bis $10 \mathrm{Uhr}$ stattfinden. Dies wird auch im Gespräch und mittels eines Aushangs in den Patientenzimmern kommuniziert. So soll sichergestellt werden, dass Aufnahme- und Entlassprozess aufeinander abgestimmt ablaufen. Durch das effektive Verweildauermanagement und der neu geschaffenen Transparenz über die geplanten Entlassungsziele für alle Berufsgruppen soll die frühzeitige Freigabe des Patientenbettes am Entlasstag und die geplante Aufnahme neuer Patienten sichergestellt werden.

Tatsächlich geht dies mit einem grundlegenden Umdenken einher: Prozesse vom Ende her zu denken. Patienten werden nach Entlassung auf Station aufgenommen (Pull-Prinzip) anstatt der gängigen
Praxis, Patienten quasi unbegrenzt auf Station zu legen und bei Kapazitätsengpässen Lösungen in einer Reihe ungeplanter Ad-hoc-Entlassungen zu suchen (Push-Prinzip) (Abb. 2).

Die Ablösung des Push-Prinzips wird je nach Notfallaufkommen der Fachabteilung im Krankenhaus kaum zu 100\% gelingen. Tatsächlich wird die Zielerreichung vielmehr in der 80\%: 20\% ParetoRegel als erfüllt gesehen, um eine deutliche Effektivitätssteigerung in der Kopplung von Aufnahmen und Entlassungen zu schaffen. Zur weiteren Erleichterung wurden Schnittstellenvereinbarungen mit der zentralen Notaufnahme und der Intensivstation (Übernahmefens-

\section{Korrespondenz}
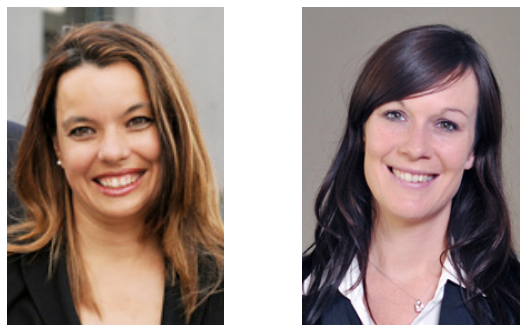

Dr. Elke Eberts Ruhl Consulting AG Katharina Ade Harrlachweg 1 68163 Mannheim Tel.: 0621/32886490 E-Mail: info@ruhl-consulting.de www.ruhl-consulting.de ter) getroffen, um die Unruhe auf Station weiter zu vermindern.

Erstaunlicherweise wurden in einem Zeitraum von nur 4 Wochen nach der Einführung des Stationskonzeptes bereits eine Verbesserung der Aufnahme- und Entlassverläufe erreicht.

Ein Jahr nach Start des Projektes und ca. 9 Monate nach Start der Umsetzungsphase war eine sichtbare Verschiebung der Entlassungen in den Vormittag und somit eine Harmonisierung der Aufnahme und Entlassungen erkennbar (Abb. 3). Dennoch bleibt dies ein Prozess, der weiterhin eng gesteuert und von allen Beteiligten immer wieder neu eingefordert werden muss. Rückschläge gehören zum Alltag, insbesondere in Situationen, in denen personelle Engpässe oder Wechsel beim ärztlichen oder pflegerischen Personal gehäuft auftreten.

\section{Fazit}

Die Effektivität und Effizienz der Prozesse auf Station ist in nicht nachrangigem Maße abhängig davon, inwieweit es gelingt, ein funktionsfähiges Managementteam aus Arzt und Pflege zu etablieren, das gemeinsam Verantwortung für die Station insgesamt und für die Einbindung der Mitarbeiter übernimmt. Eine gute Organisation der Patientenversorgung, welche durch strukturierte und abgestimmte Stationsabläufe sowie klare Führungsstrukturen ermöglicht wird, ist am Ende ausschlaggebend für die Zufriedenheit der Mitarbeiter und der Patienten.

\section{Literatur}

1 Krüttgen, N, Ruhl S. Stationskonzepte. Neuverteilung der Aufgaben- und Kompetenzprofile. klinikarzt 2012; 42: 69-71

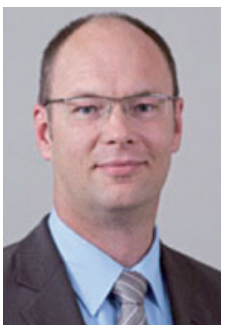

Jens Alberti Klinikum Gütersloh gGmbH Reckenberger Straße 19 33332 Gütersloh

Tel.: 05241/83 25102 E-Mail: info@klinikum-guetersloh.de www.klinikum-guetersloh.de 